\title{
Impacto del diámetro en la distribución de la presión alrededor de una elipse en un arreglo de barras cilíndricas paralelas para diferentes ángulos de inclinación respecto a un flujo de aire en un canal rectangular
}

\author{
Impact of diameter on the distribution of pressure around an ellipse \\ in an arrangement of parallel cylindrical bars for different angles of \\ inclination with respect to an air flow in a rectangular channel
}

Hernán Ferrari $^{1}$, Raúl Marino ${ }^{2}$, Víctor Herrero ${ }^{3}$

Recibido: Mayo 2021

Aceptado: Septiembre 2021

Resumen.- Se realizó el estudio experimental de la distribución de presión alrededor de un obstáculo formado por cuatro tubos cilíndricos de tres diámetros diferentes inmerso en un flujo transversal y a distintos ángulos de inclinación respecto al flujo. Se utilizó aire a presión atmosférica con flujo máximo de $0.038 \mathrm{~kg} / \mathrm{seg}$. Para cada diámetro y separación entre cilindros, la distribución de presión alrededor del obstáculo está fuertemente influenciado por el ángulo de inclinación, pero solo marginalmente afectado por el caudal (dentro del rango de trabajo). Debido a la condición de turbulencia del flujo de aire, con un número de Reynolds en la separación entre barras de 1695 a 5100, se obtuvo una muy buena concordancia entre los resultados experimentales y un modelo basado en la velocidad media del aire en ese espacio, mejor que un modelo basado en la curvatura de las líneas de corriente.

Palabras clave: Flujo de aire, flujo transversal, obstáculos cilíndricos, distribución de presión, desprendimiento de flujo.

Summary.- The experimental study of the pressure distribution around an obstacle formed by four cylindrical tubes of three different diameters immersed in a transversal flow and at different inclination angles with respect to the flow was carried out. Air at atmospheric pressure was used with a maximum flow of $0.038 \mathrm{Kg} / \mathrm{sec}$. For each cylinder bore and spacing, the pressure distribution is strongly influenced by the angle of inclination, but only marginally affected by flow (within the working range). Due to the turbulent condition of the air flow, with a Reynolds number in the separation between bars from 1695 to 5100, a very good agreement was obtained between the experimental results and a model based on the average air speed in that space, better than a model based on the curvature of streamlines in the gap.

Keywords: Air flow, yawed flow, cylindrical obstacle, pressure distribution, flow detachment.

\footnotetext{
1 Doctor en Ciencias Físicas, Universidad Austral - CONICET (Argentina), hferrari@austral.edu.ar, ORCID iD: https://orcid.org/0000-0002-7024-3900

2 Doctor en Ingeniería, Facultad de Ciencias Exactas y Naturales, Universidad Nacional de Cuyo (Argentina), rmarino@fcen.uncu.edu.ar, ORCID iD: https://orcid.org/0000-0003-2654-3583

3 Doctor en Ingeniería Nuclear, Universidad Austral - LIDTUA (Argentina), vherrero@ austral.edu.ar, ORCID iD: https://orcid.org/0000-0001-9013-2135
} 
1. Introducción. - El comportamiento de un fluido al atravesar obstáculos con forma de cilindros es de suma importancia en aplicaciones de ingeniería tales como intercambiadores de calor, reactores nucleares, procesos químicos, estructuras civiles, etc. generando en esta área de conocimiento un problema tan interesante y complejo que ha motivado una gran cantidad de estudios experimentales y numéricos realizados por un importante número de investigadores. El entendimiento del flujo alrededor de distintas formas de obstáculos, su orientación y distancia relativa es necesaria para las aplicaciones mencionadas y muchas otras. Las características del flujo respecto al espaciamiento entre cilindros en distintos arreglos conducen a una transición de un régimen laminar a uno turbulento con la aparición de vórtices en el flujo [1-4], siendo el número de Reynolds un indicativo de la transición. Los estudios en arreglos de bancos de tubos en un flujo transversal han mostrado que no solamente tienen impacto en la pérdida de carga, sino que también producen efectos en la eficiencia del intercambio de calor [5], siendo esto de gran relevancia, por ejemplo, en el núcleo de un reactor nuclear, observándose diferentes resultados respecto tanto del ángulo de incidencia como del número de Reynolds [6]. Para el caso de intercambiadores que utilizan aire como fluido, tanto la geometría como el ángulo de incidencia del fluido respecto de los obstáculos son de suma importancia [7].

Se ha demostrado que a distintos ángulos de incidencia y números de Reynolds se verifica el principio de independencia al estudiar ciertos comportamientos para ángulos entre $40^{\circ} \mathrm{Y} 50^{\circ}$ [8]. Schewe encontró que, en el caso de reactores nucleares, el coeficiente de arrastre y la frecuencia de desprendimiento de vórtices no son sensibles al número de Reynolds dentro del régimen subcrítico $(300<\operatorname{Re}<3000)$ [9]. En el artículo de Zhao[10] puede encontrarse una revisión actualizada donde se presentan varios cálculos numéricos del flujo cruzado alrededor de los cilindros en geometrías $2 \mathrm{D}$ y $3 \mathrm{D}$.

Habiéndose estudiado tanto experimental como numéricamente el flujo que atraviesa en forma oblicua obstáculos cilíndricos [10-17], se ha observado con técnicas de visualización que el flujo muestra una fuerte dependencia con el número de Reynolds en la zona cercana a los obstáculos, pero sin efectos significativos en promedio en las zonas periódicas [18].

Al utilizar una configuración con obstáculos en forma de barras cilíndricas se observó que la distancia entre las mismas produce efectos en la dinámica del fluido que puede inducir vibraciones y así afectar la estabilidad de la estructura. De la misma manera, en la literatura se mencionan el estudio de las interacciones y simetrías en el flujo cuando dos barras están lo suficientemente cerca, identificando regiones donde pueden ocurrir separación de burbujas [19].

En este trabajo se presentan los estudios experimentales y su comparación con modelos teóricos del coeficiente de presión en la pared $(\mathrm{Cp})$ alrededor de un obstáculo cilíndrico en un arreglo periódico para diferentes ángulos de inclinación respecto del flujo que lo atraviesa, como así también su dependencia del diámetro de las barras y la separación entre los obstáculos. En estudios anteriores se analizaron configuraciones similares [20] sin el estudio de la dependencia para distintos diámetros y espaciamientos entre cilindros, mostrando en este trabajo incluso algunas diferencias con los resultados obtenidos en el pasado.

2. Método y dispositivo experimental. - El dispositivo experimental, que puede verse en la fotografía de la Figura I, es similar al utilizado en estudios anteriores utilizando un canal rectangular por el cual fluye aire inyectado por un soplador axial.

El flujo es homogeneizado por un conjunto de tres mallas metálicas que se encuentran en la zona de ingreso del canal y que se encuentra a la derecha de la región central del dispositivo que se muestra en la Figura I, donde la uniformidad del perfil de velocidades se verificó midiendo los valores locales con un conjunto de anemómetros de hilo caliente. En la zona central se encuentran las barras cilíndricas las cuales pueden orientarse en diferentes ángulos cambiando la posición del panel circular móvil que se muestra en la Figura II.

Memoria Investigaciones en Ingeniería, núm. 21 (2021). pp. 56-70

https://doi.org/10.36561/ING.21.6

ISSN 2301-1092・ISSN (en línea) 2301-1106 


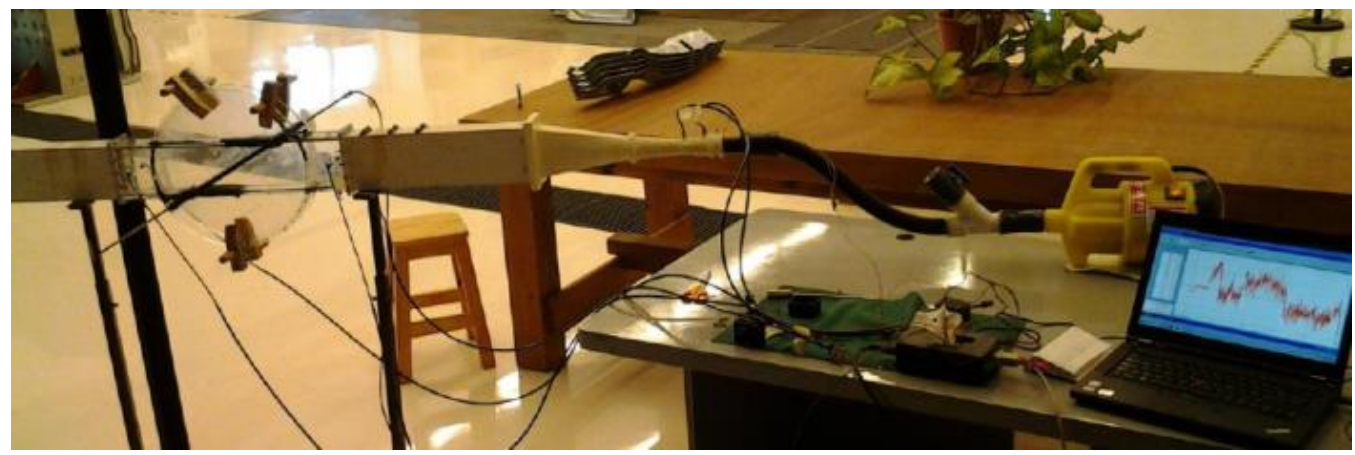

Figura I. Fotografía del dispositivo experimental.

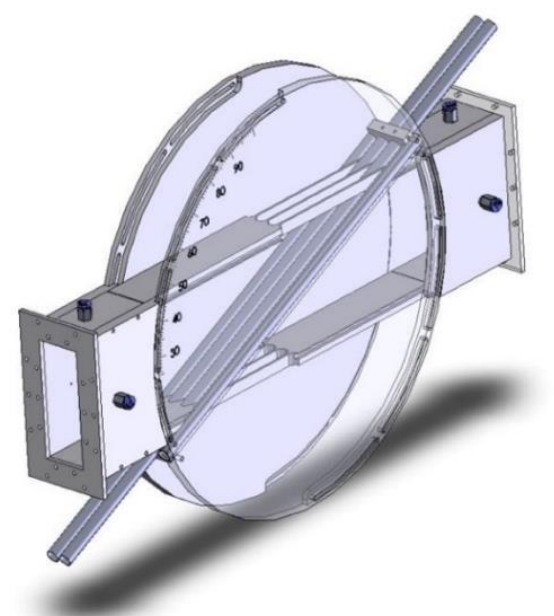

Figura II. Arreglo experimental. Región de prueba

El flujo en la sección de prueba (Figura II) es forzado a pasar a través de un obstáculo formado por cuatro barras cilíndricas, dos de ellas en la parte central y dos semibarras en las paredes del canal rectangular. A diferencia de estudios anteriores, se utilizaron tres conjuntos de cuatro barras con diámetros de $10.86 \mathrm{~mm}, 8.3 \mathrm{~mm}$ y $6.50 \mathrm{~mm}$ con la consiguiente separación entre ellas de $2 \mathrm{~mm}$, $4.56 \mathrm{~mm}$ y $6.36 \mathrm{~mm}$ respectivamente. La inclinación de las barras respecto del flujo se mide con un goniómetro en la parte del disco móvil que permite una precisión de medio grado. La sección de salida evita la interferencia aguas abajo en el flujo de la sección de prueba y proporciona una ubicación conveniente para la medición de las condiciones de salida. El caudal se mide con un tubo Venturi (Figura III) ubicado antes de la entrada a la región de prueba. El Venturi está conectado a un sensor de presión diferencial DP Honeywell modelo SCX05 y también se colocó un sensor de temperatura RTD para controlar la temperatura del aire durante el experimento.

Las dimensiones de la sección de prueba son:

- Largo del canal: $500 \mathrm{~mm}$

- Ancho del canal: $38.58 \mathrm{~mm}$

- Altura del canal: $100 \mathrm{~mm}$
Coordenada $\mathrm{x}$

Coordenada y

Coordenada $\mathrm{z}$

Memoria Investigaciones en Ingeniería, núm. 21 (2021). pp. 56-70

https://doi.org/10.36561/ING.21.6 
Las dimensiones de los canales son:

- Largo del canal de ingreso: $400 \mathrm{~mm}$

- Largo del canal de salida: $600 \mathrm{~mm}$

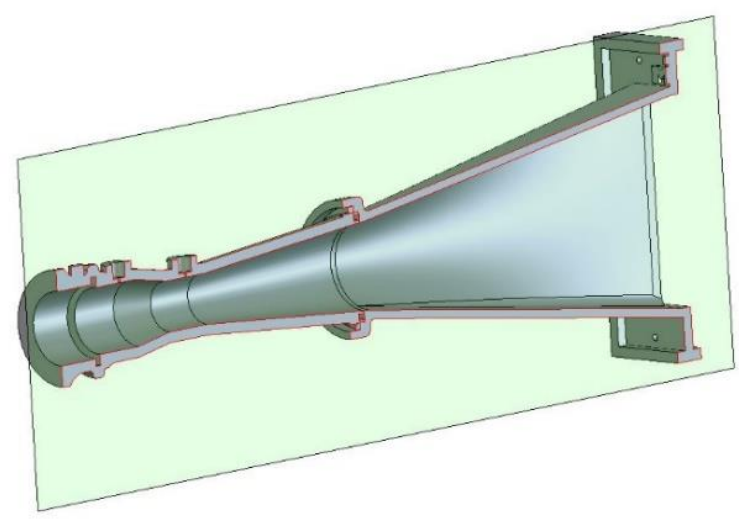

Figura III. Tubo de Venturi

En una de las dos barras centrales se realizó un orificio de $0.5 \mathrm{~mm}$ de diámetro en la posición del punto medio vertical, para medir la presión de pared al hacer girar manualmente la barra sobre su eje. Utilizando otro sensor similar de presión se midió la diferencia entre la presión en la superficie de la barra y la presión global en la sección de entrada.

Una vez alcanzada la estabilización de la temperatura del aire que ingresa con caudal constante en el canal, se registró la diferencia de presión entre la pared de la barra y la entrada para diferentes ángulos $\theta$, de giro del punto de medición de la presión en un plano normal al eje de la barra (siendo $\theta=0$ la dirección del flujo) (Figura IV), utilizando un goniómetro solidario a uno de los extremos de la barra. La inclinación de los obstáculos (barras) se fijó a ángulos $\alpha=30^{\circ}, 60^{\circ}$ y $90^{\circ}$ con respecto a la dirección del flujo.

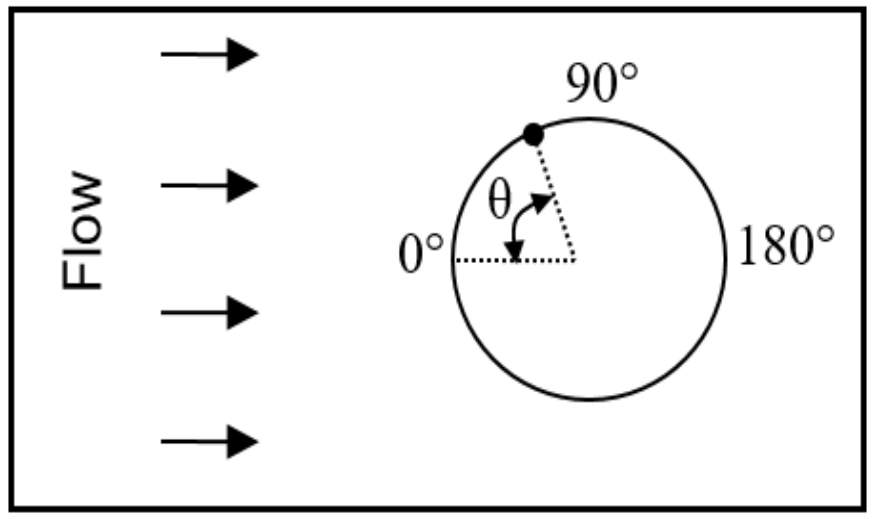

Figura IV. - Definición del ángulo de medición en coordenadas polares en la barra central.

3. Resultados. - En las Figuras V a VII se muestran las dependencias en $\theta$, tomando el diámetro de las barras como parámetro, para ángulos $\alpha$ de inclinación de $30^{\circ}, 60^{\circ}$ y $90^{\circ}$ en cada figura respectivamente. 
Se define el coeficiente de presión de pared como (Fórmula I):

$$
C_{P}(\theta)=\frac{P_{w}(\theta)-P_{\infty}}{\frac{1}{2} \rho v_{\infty}^{2}}
$$

en donde $\rho$ simboliza la densidad del gas, $P_{w}(\theta)$ es la presión de pared en función del ángulo $\theta$, y $P_{\infty}$ y $v_{\infty}$ corresponden a la presión y velocidad de entrada al canal, $v_{\infty}=\frac{\dot{m}}{\rho_{\infty} A_{\infty}} \operatorname{con} \dot{m}$ la tasa de flujo másico y $\rho_{\infty}$ y $A_{\infty}$ la densidad del aire y el área de la sección de ingreso. Cada figura corresponde a mediciones realizadas para un ángulo $\alpha$ constante. Dado que se verificó que las mediciones eran simétricas para ángulos $180^{\circ} \leq \theta \leq 360^{\circ}$, solamente se muestran los resultados en el rango $0^{\circ} \leq \theta \leq 180^{\circ}$.

El diámetro hidráulico del sistema se calcula como $D_{h}=\frac{2}{\left(h^{-1}+\varepsilon^{-1}\right)}$, en donde $h$ corresponde a la altura del canal de medición y $\varepsilon$ es el espacio entre las barras que es función del diámetro de las mismas al ser el ancho del canal fijo. Los números de Reynolds en el espacio entre barras calculado como $R e=\frac{v_{\infty} D_{h}}{v}$ resulta en valores de $R e=1695$ para d $=10.86 \mathrm{~mm} ; R e=3770$ para $\mathrm{d}=$ $8.3 \mathrm{~mm}$ y $R e=5100$ para $\mathrm{d}=6.5 \mathrm{~mm}$.

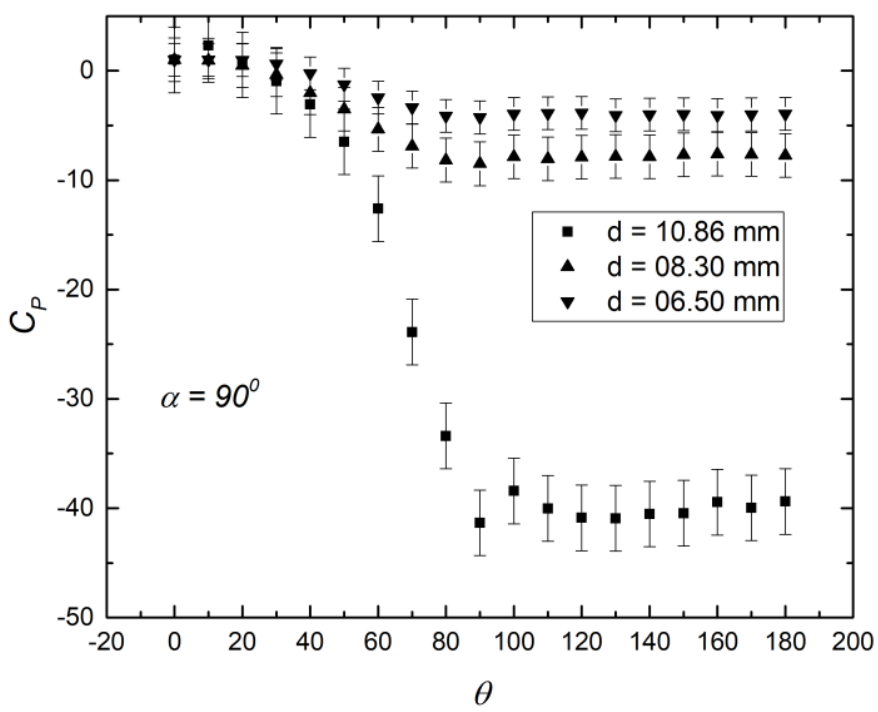

Figura V. Perfiles polares del coeficiente de presión para el arreglo de barras de distintos diámetros formando un ángulo de 90 grados respecto del flujo de aire. 


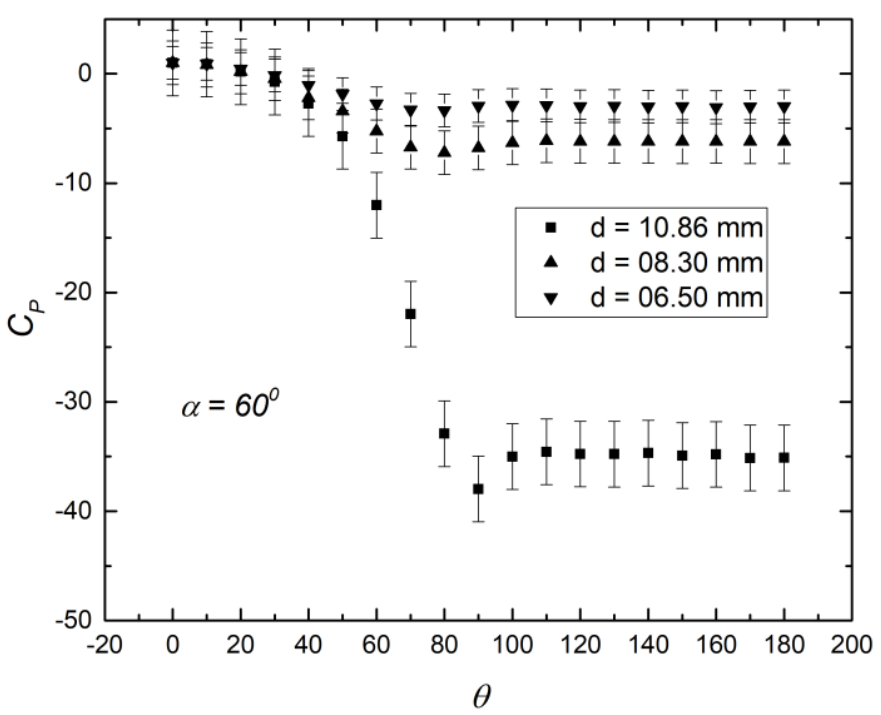

Figura VI. Perfiles polares del coeficiente de presión para el arreglo de barras de distintos diámetros formando un ángulo de 60 grados respecto del flujo de aire.

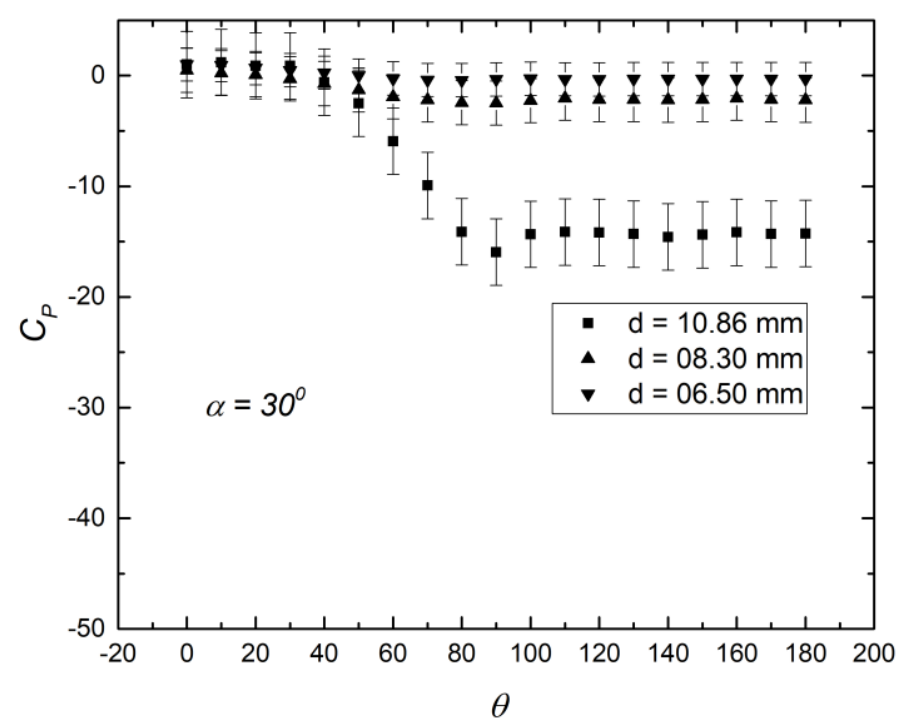

Figura VII. Perfiles polares del coeficiente de presión para el arreglo de barras de distintos diámetros formando un ángulo de 30 grados respecto del flujo de aire. 
Las Figuras VIII a X muestran el coeficiente de la presión de pared $C_{P}$ y su dependencia con el ángulo $\theta$, dejando fijo en cada caso el diámetro $d=10.86 \mathrm{~mm} ; 08.30 \mathrm{~mm}$ y $06.50 \mathrm{~mm}$ y tomando como parámetro los tres diferentes ángulos de inclinación de las barras respecto del flujo en el canal.

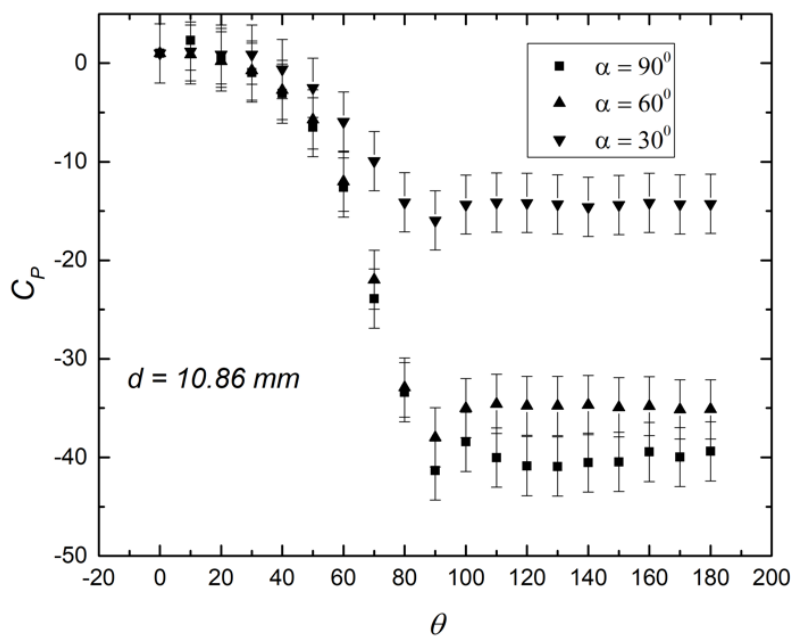

Figura VIII. Perfiles polares del coeficiente de presión para el diámetro d $=10.86 \mathrm{~mm}$ y tres diferentes ángulos de inclinación del arreglo de barras $\left(\alpha: 30^{\circ} ; 60^{\circ} ; 90^{\circ}\right)$.

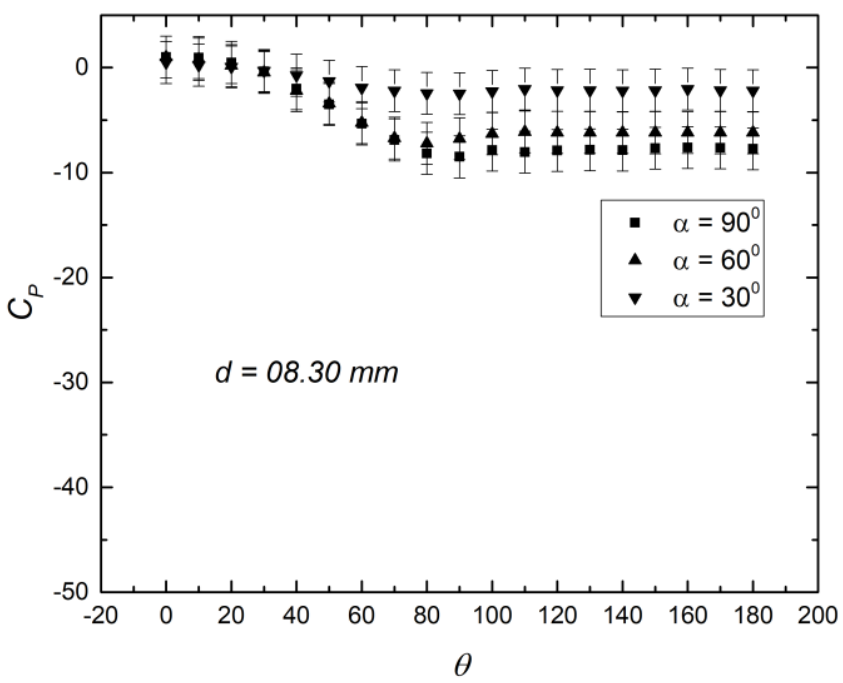

Figura IX. Perfiles polares del coeficiente de presión para el diámetro d $=08.30 \mathrm{~mm}$ y tres diferentes ángulos de inclinación del arreglo de barras $\left(\alpha: 30^{\circ} ; 60^{\circ} ; 90^{\circ}\right)$. 


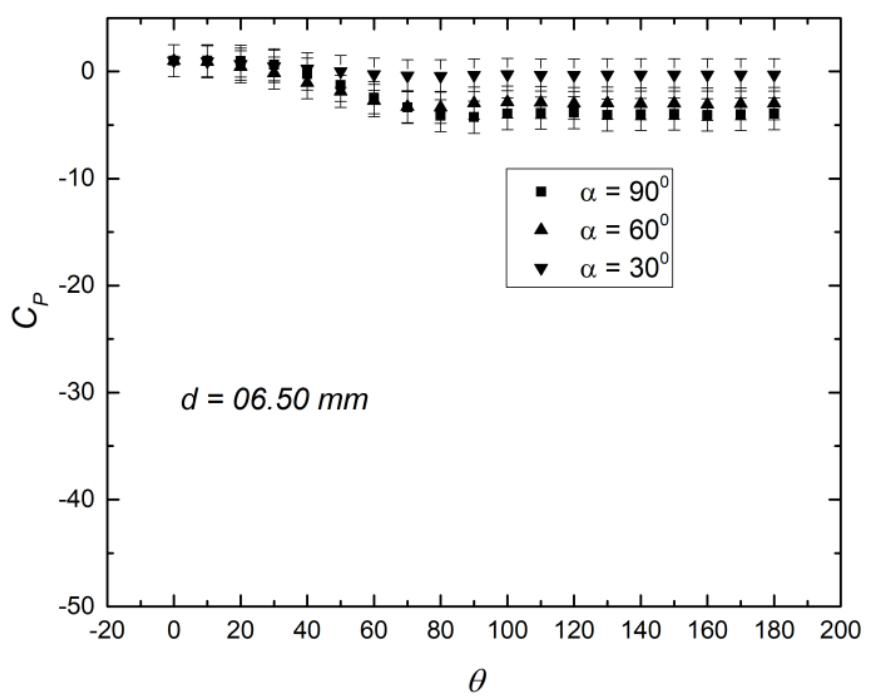

Figura X. Perfiles polares del coeficiente de presión para el diámetro d $=06.50 \mathrm{~mm}$ y tres diferentes ángulos de inclinación del arreglo de barras $\left(\alpha: 30^{\circ} ; 60^{\circ} ; 90^{\circ}\right)$.

Se puede observar en los resultados obtenidos, dentro del error experimental, que el $C_{P}$ es independiente de la tasa de flujo utilizada en los experimentos[21]. La menor presión se obtiene siempre para el ángulo $\theta=90^{\circ}$. Para ángulos $\theta>90^{\circ}$ se observa una suave recuperación de la presión y luego la presión se mantiene constante hasta $\theta=180^{\circ}$, lo cual es un indicador de la falta de desarrollo del flujo para estos ángulos. Para $\alpha=90^{\circ}$ la recuperación de la presión ocurre hasta aproximadamente $\theta=110^{\circ}$, más cercano a $\theta=90^{\circ}$ que en un flujo que atraviesa una única barra aislada [22]. Esto sugiere que la presencia de barras cercanas dificulta el desarrollo del flujo con un consecuente retraso en la recuperación de la presión.

A medida que el arreglo de barras aumenta su inclinación respecto del flujo de aire (el valor de $\alpha$ decrece), los valores de presión mínima a $\theta=90^{\circ}$ y la presión en la parte trasera de la barra a $180^{\circ}$ aumenta. De la misma forma el ángulo de interrupción de la presión se mueve progresivamente a ángulos mayores, alcanzando un valor de $\theta=140^{\circ}$ para $\alpha=30^{\circ}$. Estos efectos son razonables ya que la forma de la intersección de las barras con un plano xy tiende a elipses que son mejores por su aerodinámica cuánto más largo es el eje paralelo al flujo Figura XI. 


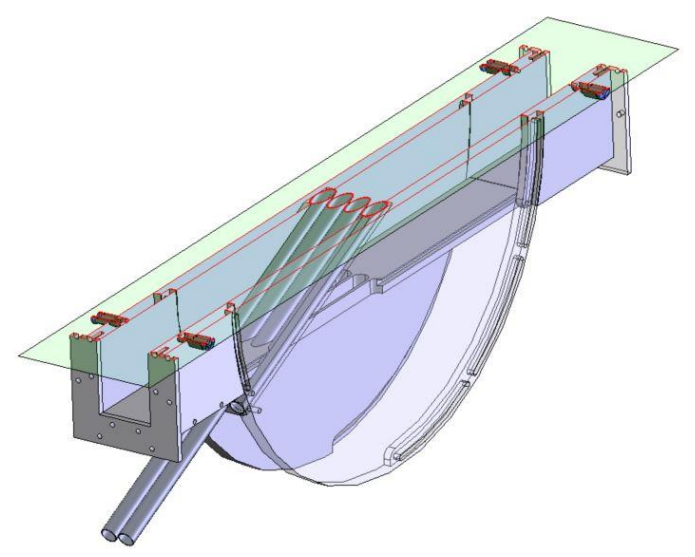

Figura XI. Visualización de las elipses formadas por la intersección de las barras con el plano xy a $z=0$, paralelo al flujo de aire

La Figura XII muestra la presión adimensional de la pared trasera promediada entre los caudales medidos para diferentes ángulos de inclinación y para diferentes diámetros de barras. Las correlaciones correspondientes para cada diámetro son:

$$
\begin{aligned}
& C_{P} 10.86=(-0.47 \pm 0.13) \alpha \pm(-0.5 \pm 0.5) \\
& C_{P} 08.30=(-0.10 \pm 0.02) \alpha \pm(0.8 \pm 0.8) \\
& C_{P} 06.50=(-0.07 \pm 0.01) \alpha \pm(1.8 \pm 0.4)
\end{aligned}
$$

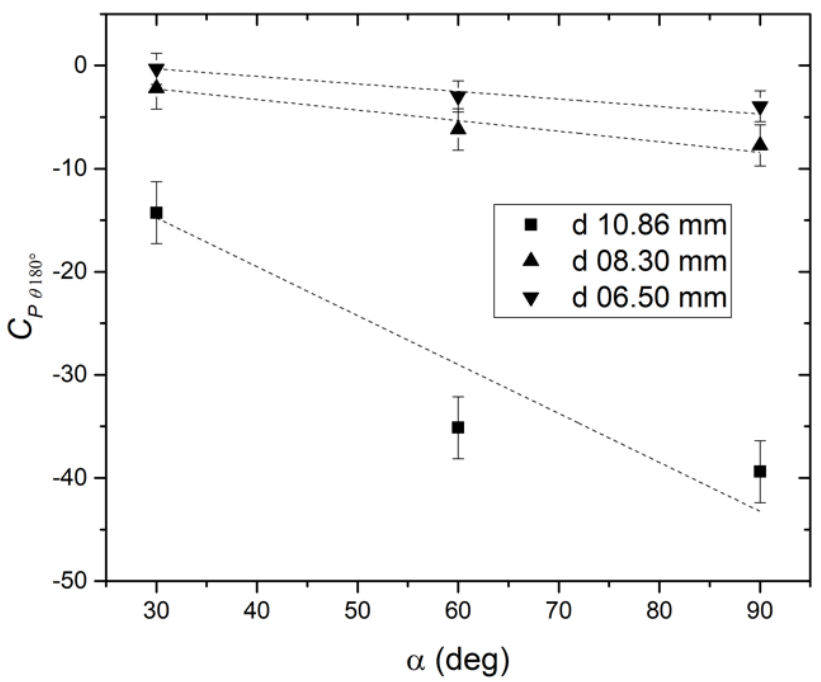

Figura XII. Dependencia del coeficiente de presión en la región trasera de la barra para diferentes diámetros y ángulos de inclinación de las barras. 
En el presente trabajo, para el caso específico del arreglo de barras de diámetro $d=10.86 \mathrm{~mm}$, se obtuvieron valores en las correlaciones de $\bar{C}_{D} 10.86$ que difieren de los obtenidos por Marino [21]. En ese caso la tasa de flujo másico fue de $0.0825 \mathrm{~kg} / \mathrm{s}$. En la Figura XIII se comparan estas diferencias. Esto puede deberse a que el flujo entre las barras presenta un perfil plano, lo que significa una velocidad uniforme.

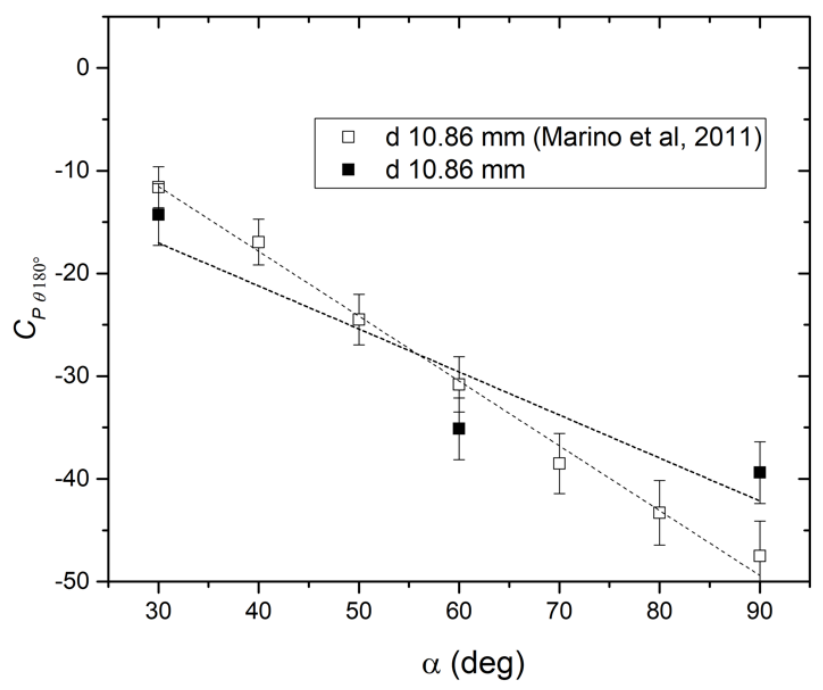

Figura XIII. Comparación para el mismo arreglo de barras de diámetro d=10.86 a una tasa de flujo másico $\dot{m}=0.0825 \mathrm{~kg} / \mathrm{s}$ (Marino[21]) y una tasa de $\dot{m}=0.031 \mathrm{~kg} / \mathrm{s}$

4. Modelo y análisis teórico. - Se desarrolló un modelo teórico del comportamiento del sistema en la separación entre barras, para comparar contra los resultados experimentales obtenidos para el caso del flujo perpendicular al arreglo de barras $\left(\alpha=90^{\circ}\right)$. En este modelo se desarrolló un caso de flujo bidimensional y ciertas simplificaciones para poder obtener el perfil del coeficiente de presión $C_{P}$.

4.1. Presión de pared en la separación entre barras a $\alpha=90^{\circ}$. - Se calculó la presión de pared a $\alpha=90^{\circ}$ considerando el caso de un arreglo de barras cilíndricas infinitas perpendiculares al flujo de aire. Para ello se analizó el balance de fuerzas en un pequeño volumen de control y los gradientes de presiones normales a las líneas de corrientes en un flujo bidimensional dado por Shapiro [23]:

$$
\frac{\partial p}{\partial n}=\frac{\rho v^{2}}{R}
$$

en donde R corresponde a la curvatura de las líneas de corriente.

Por simplicidad colocaremos el origen del sistema de coordenadas en el punto medio entre las dos barras centrales. De la Ecuación II utilizando la coordenada "y" que pasa por el centro de los cilindros y normal a las líneas de corriente determina la siguiente dependencia de la presión Figura XIV. 


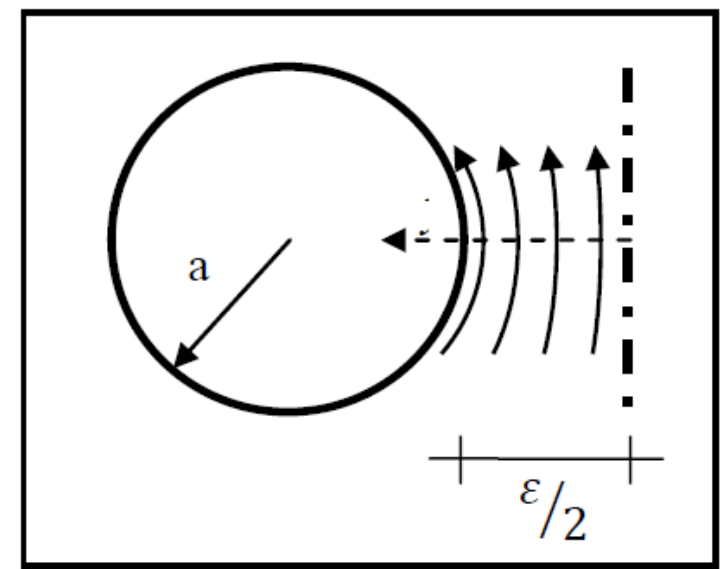

Figura XIV. Diagrama con las variables involucradas y sistema de referencia. $A=$ radio del cilindro y $\varepsilon=$ separación entre las barras

$$
\frac{\partial p}{\partial n}=\frac{\rho v^{2}}{R(y)}
$$

El radio de curvatura de las líneas de corriente $\mathrm{R}(\mathrm{y})$ es infinito en el punto central del espaciado entre dos barras $(y=0)$, mientras que la curvatura en la pared de las barras es igual al radio de los cilindros $\left(y=\frac{\varepsilon}{2}\right)$. Asumiendo una variación lineal de los radios de curvatura de las líneas de corriente y una velocidad promedio constante en el espaciado entre barras, se puede integrar la Ecuación III para obtener la diferencia de presiones entre el punto medio del espaciado $(y=0)$ y la pared $\left(y=\frac{\varepsilon}{2}\right)$. El resultado que se obtiene es:

$$
P_{(0)}-P_{w}=\frac{\varepsilon}{4 a} \rho \bar{v}^{2}
$$

En donde la velocidad promedio en el espaciado $\bar{v}$, satisface:

$$
\bar{v}=v_{\infty} \frac{A_{\infty}}{A_{g}}
$$

Donde $A_{\infty}$ corresponde a la sección transversal de la región del canal libre de obstáculos y $A_{g}$ corresponde al área mínima entre barras.

Utilizando la ecuación de Bernoulli:

$$
P_{\infty}+\frac{1}{2} \rho v_{\infty}^{2}=P_{(0)}+\frac{1}{2} \rho \bar{v}^{2}
$$

Podemos obtener de esta forma usando la Ecuación I el coeficiente de presión a $\theta=90^{\circ}$ :

$$
C_{P}\left(\theta=90^{\circ}, \alpha=90^{\circ}\right)=1-\left(1+\frac{\varepsilon}{2 a}\right)\left(\frac{A_{\infty}}{A_{g}}\right)^{2}
$$

Cuyo resultado $C_{P}\left(90^{\circ}\right)=-47.95$ es muy cercano al obtenido experimentalmente $(-45.8 \pm 2.1)$. 
4.2. Presión de pared en la separación entre barras a $\alpha<90^{\circ}$. - Dado que la sección transversal de una barra cilíndrica inclinada es una elipse con el semieje menor $a$ y el semieje mayor $\frac{a}{\sin (\alpha)}$, el modelo anterior puede extenderse para $30^{\circ} \leq \alpha \leq 90^{\circ}$, tomando el radio de curvatura en la pared de la barra $\left(y=\frac{\varepsilon}{2}\right)$ como $\frac{a}{\sin ^{2}(\alpha)}$ para $\theta=90^{\circ}$. De la misma forma, el área mínima que resulta en la región entre barras, $A_{g}$, aumenta en un factor $\frac{1}{\sin (\alpha)}$, debido a la inclinación de las barras. Aproximando el flujo en el espacio entre barras circulares inclinadas, como el flujo en el espacio entre barras elípticas, perpendiculares al flujo, la Ecuación VII se puede extender a:

$$
C_{P}\left(\theta=90^{\circ}, \alpha\right)=1-\sin ^{2}(\alpha)\left(1+\frac{\varepsilon}{2 a} \sin ^{2}(\alpha)\right)\left(\frac{A_{\infty}}{A_{g}}\right)^{2}
$$

En las Figuras XV a XVIII se muestra la dependencia del coeficiente de presión de pared $C_{D}\left(\theta=90^{\circ}\right)$ con el ángulo de inclinación entre el flujo de aire y el arreglo de barras, $\alpha$, para $d=$ $10.86 \mathrm{~mm} ; 08.30 \mathrm{~mm}$ and $06.50 \mathrm{~mm}$ y su comparación con los resultados obtenidos con el modelo descripto.

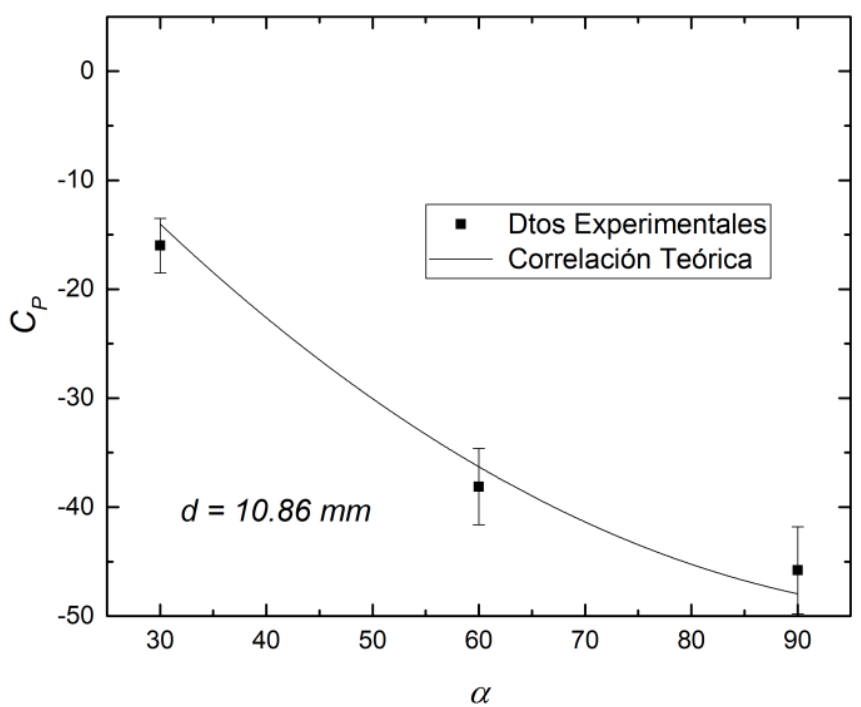

Figura XV. $C_{P}\left(\theta=90^{\circ}\right)$ para un diámetro de barras $d=10.86 \mathrm{~mm}$ y ángulo de inclinación $\alpha$ $\left(30^{\circ} ; 60^{0} ; 90^{\circ}\right)$. Correlación entre resultados experimentales y del modelo teórico. 


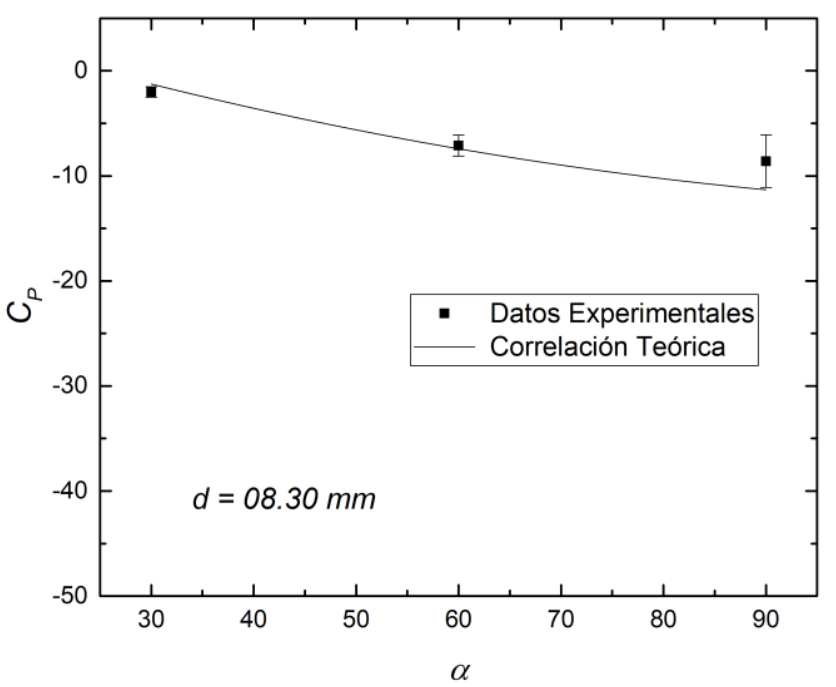

Figura XVI. $C_{P}\left(\theta=90^{\circ}\right)$ para un diámetro de barras $d=08.30 \mathrm{~mm}$ y ángulo de inclinación $\alpha\left(30^{\circ} ; 60^{0} ; 90^{\circ}\right)$. Correlación entre resultados experimentales y del modelo teórico.

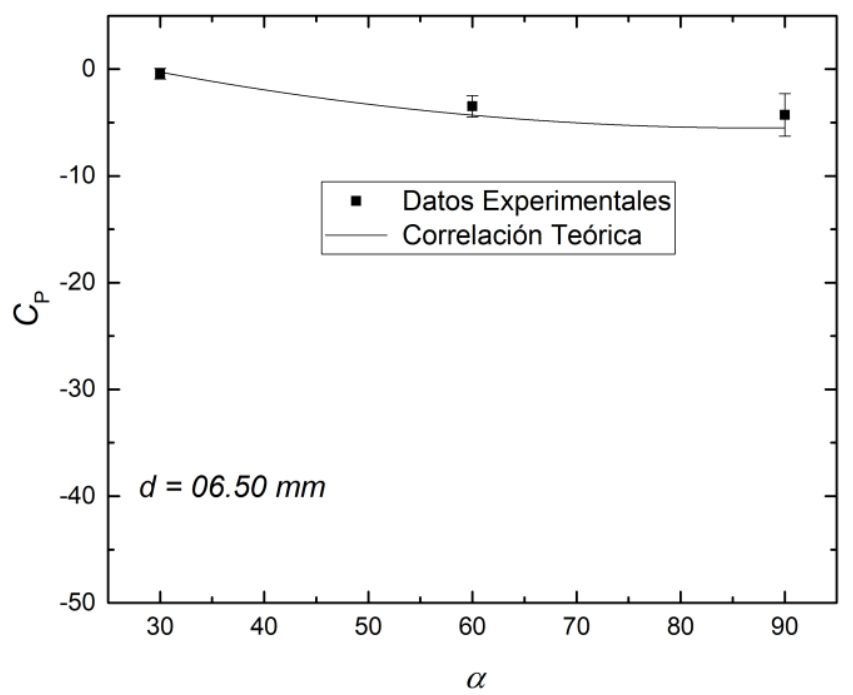

Figura XVII. $C_{P}\left(\theta=90^{\circ}\right)$ para un diámetro de barras $d=06.50 \mathrm{~mm}$ y ángulo de inclinación $\alpha\left(30^{0} ; 60^{0} ; 90^{\circ}\right)$. Correlación entre resultados experimentales y del modelo teórico. 


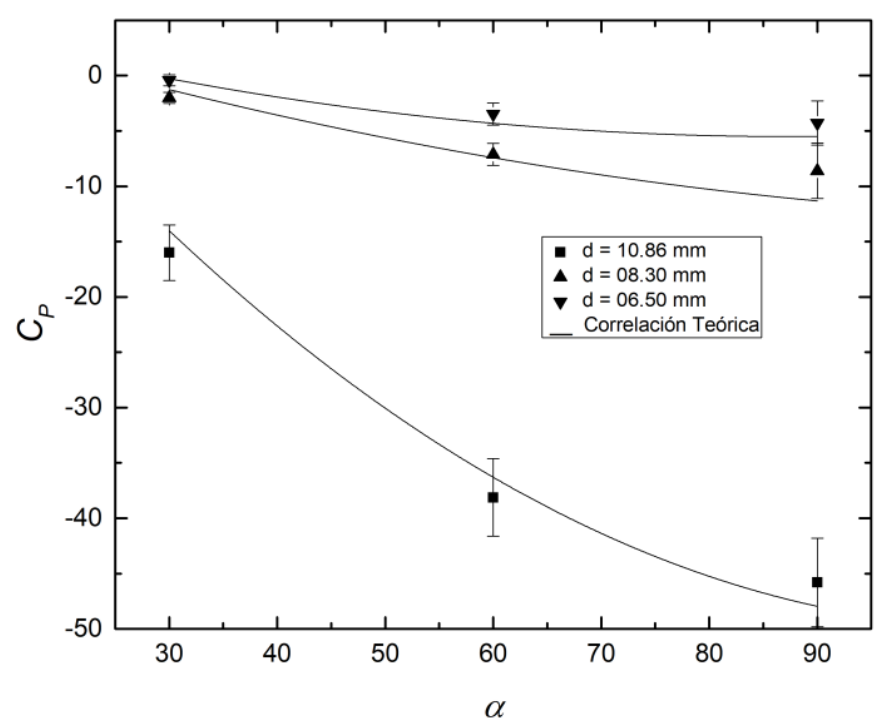

Figura XVIII. $C_{P}\left(\theta=90^{\circ}\right)$ para los tres diámetros de barras y ángulo de inclinación $\alpha$ $\left(30^{\circ} ; 60^{\circ} ; 90^{\circ}\right)$. Correlación entre resultados experimentales y del modelo teórico.

5. Conclusiones. - Se estudió experimentalmente la distribución de la presión de pared para un flujo de aire que atraviesa un arreglo de barras cilíndricas para tres diferentes diámetros e inclinaciones de las barras respecto al flujo de aire. Los resultados obtenidos muestran una fuerte dependencia de los coeficientes con la inclinación de las barras no así con la tasa de flujo másico (con valores de $0.038 \mathrm{~kg} / \mathrm{seg} ; 0.030 \mathrm{~kg} / \mathrm{seg} ; 0.026 \mathrm{~kg} / \mathrm{seg}$ ). Pudo observarse experimentalmente que el coeficiente de presión de pared depende fuertemente del diámetro de las barras y que, a pesar de que a menor diámetro mayor es la separación entre barras, el retraso en el desarrollo del flujo detrás del obstáculo sigue estando presente en todas las condiciones experimentales, produciéndose en todos los casos una interrupción de la recuperación de la presión a partir de un ángulo $\theta=100^{\circ}$.

Para los diferentes espacios entre barras analizados y las condiciones de turbulencia para el flujo de aire con números de Reynolds en el espacio entre barras entre 1695 y 5100, se analizó un modelo teórico considerando una velocidad promedio en el espaciado entre barras obteniéndose un acuerdo entre este modelo y los resultados experimentales obtenidos dentro de un error del $10 \%$ acumulado entre las mediciones de todas las variables.

La inclinación de las barras cilíndricas permite aumentar la superficie de contacto con el fluido disminuyendo al mismo tiempo la pérdida de presión por la presencia del obstáculo. El modelo teórico permite calcular el coeficiente de presión para un ángulo $\theta=90^{\circ}$, aplicación de gran utilidad, por ejemplo, en intercambiadores de calor. 


\section{Referencias. -}

[1] Zdravkovich, M., Flow Around Circular Cylinders: 1 Fundamentals, 2 Applications, Oxford University Press, 1997.

[2] Bloor, M.S., "The transition to turbulence in the wake of a circular cylinder". J Fluid Mech 19(2). pp. 290. 1964.

[3] Gerrard J., "The mechanics of the formation region of vortices behind bluff bodies". J Fluid Mech 25(2). pp. 401. 1966.

[4] Roshko A., "Experiments on the flow past a circular cylinder at very high Reynolds number". J Fluid Mech 10(3). pp. 345. 1961.

[5] Seong-Yeon Y., Hwa-Kil K., Jin-Hyuk K., "A study on heat transfer characteristics for staggered tube banks in cross flow", Journal of mechanical science and technology, 21. pp. 505. 2007.

[6] R. Marino, V. Herrero, N. Silin, J. Converti, A. Clausse. "Polar Profile of the Wall Pressure on Cylindrical Bars in Yawed Gas Flow”, Latin American Applied Research, 40(3). pp. 287. 2010.

[7] Meyer, C. J., Kröger, D. G., "Air-cooled heat exchanger inlet flow losses”, Applied thermal Engineering, 21. pp. 771. 2001.

[8] Vakil, A., Green, S., "Drag and lift coefficients of inclined finite circular cylinders at moderate Reynolds numbers", Computer \& Fluids, 38. pp. 1771. 2009.

[9] Schewe, G., "On the force fluctuations acting on a circular cylinder in cross-flow from subcritical up to transcritical Reynolds numbers", J. Fluid Mech., 133. pp 265. 1983.

[10] Zhao, M., Cheng, L. and Zhou T., "Direct numerical simulation of three-dimensional flow past a yawed circular cylinder of infinite length", J. Fluids Structures, 25. pp. 831. 2009.

[11] King, R., "Vortex excited oscillations of yawed circular cylinders", ASME J. Fluids Eng., 99. pp. 495.1977.

[12] Thakur, A., Liu, X. and Marshall, J., "Wake flow of single and multiple yawed cylinders", ASME J. Fluids Eng., 126. pp. 861. 2004.

[13] Chiba, K. and Horikawa, A., "Numerical solution for the flow of viscoelastic fluids around an inclined circular cylinder. II. The hydrodynamic force on a circular cylinder", Rheological Acta, 26. pp. 255. 1987.

[14] Marshall, J., "Wake dynamics of a yawed cylinder", ASME J. Fluids Eng. 125. pp. 97. 2003. [15] Lucor, D. and Karniadakis G., "Effects of oblique inflow in vortex-induced vibrations", Flow, Turbulence and Combustion, 71. pp. 375. 2003.

[16] Kim, T., Hodson, H. and Lu T., "On the prediction of pressure drop across banks of inclined cylinders", Int. J. Heat Fluid Flow, 27. pp. 311. 2006.

[17] Fowler, A. and Bejan, A., "Forced convection in banks of inclined cylinders at low Reynolds numbers", Int. J. Heat Fluid Flow, 15. pp. 90. 1994.

[18] Paul, S.S., Tachie, M.F., Ormiston, S.J., "Experimental study of turbulent cross-flow in a staggered tube bundle using particle image velocimetry", international Journal of Heat and Fluid Flow, 28. pp. 441. 2007.

[19] Alam, Md. M., Zhou, Y., "Flow around two side-by-side closely spaced circular cylinders", Journal of fluid and structures, 23. pp. 799. 2007.

[20] Peybernès, J., "Evaluation of the forces generated by cross-flow on PWR fuel assembly", IAEA-TECDOC, 13. pp. 1454. 2005.

[21] Marino, R., Clausse, A., Herrero, V., Silin, N., Saravia, G., "Wall pressure profile around cylindrical rods in yawed gas flow", Journal of fluid engineering, 133(7). 074502. 2011.

[22] White, F. M., Fluid mechanics, McGraw-Hill, 3rd Ed., Mexico, 1996.

[23] Shapiro, A., The dynamics and thermodynamics of compressible flow, The Ronald Press Co., New York, 1953. 